\title{
Astrophysical relevance of the low-energy dipole strength of ${ }^{206} \mathrm{~Pb}$
}

\author{
A.P. Tonchev ${ }^{1,2}$, N. Tsoneva ${ }^{3}$, S. Goriely ${ }^{4}$, C. Bhatia ${ }^{2,5}$, C.W. Arnold ${ }^{6}$, S.L. Hammond ${ }^{7,5}$, J.H. Kelley ${ }^{8,5}$, E. Kwan ${ }^{9}$, \\ H. Lenske ${ }^{3}$, J. Piekarewicz ${ }^{10}$, R. Raut ${ }^{11}$, G. Rusev ${ }^{6}$, T. Shizuma ${ }^{12}$, and W. Tornow ${ }^{2,5}$ \\ ${ }^{1}$ Nuclear and Chemical Sciences Division, Lawrence Livermore National Laboratory, Livermore, California 94550, USA \\ ${ }^{2}$ Duke University, Department of Physics, Box 90308, Durham, North Carolina 27708-0308, USA \\ ${ }^{3}$ Institut für Theoretische Physik, Universität Gießen, Gießen, D-35392, Germany \\ ${ }^{4}$ Institut d'Astronomie et d'Astrophysique, Université Libre de Bruxelles, Campus de la Plaine, CP-226, 1050 Brussels, Belgium \\ ${ }^{5}$ Triangle Universities Nuclear Laboratory, Durham, North Carolina 27708-0308, USA \\ ${ }^{6}$ Chemistry Division, Los Alamos National Laboratory, Los Alamos, New Mexico 87545, USA \\ ${ }^{7}$ Department of Physics and Astronomy, University of North Carolina at Chapel Hill, Chapel Hill, North Carolina 27599-3255, USA \\ ${ }^{8}$ North Carolina State University, Department of Physics, Box 8202, Raleigh, North Carolina 27695-8202, USA \\ ${ }^{9}$ National Superconducting Cyclotron Laboratory, East Lansing, Michigan 48824, USA \\ ${ }^{10}$ Department of Physics, Florida State University, Tallahassee, Florida 32306-4350, USA \\ ${ }^{11}$ UGC-DAE Consortium for Scientic Research, Kolkata Centre LB-8 Sector-III, Bidhannagar, Kolkata 700098, India \\ ${ }^{12}$ Quantum Beam Science Center, Japan Atomic Energy Agency, Tokaimura, Ibaraki 319-1184, Japan
}

\begin{abstract}
The dipole strength of ${ }^{206} \mathrm{~Pb}$ was studied below the neutron separation energy using photon scattering experiments at the HIGS facility. Utilizing the technique of nuclear resonance fluorescence with $100 \%$ linearly-polarized photon beams, the spins, parities, branching ratios and decay widths of excited states in ${ }^{206} \mathrm{~Pb}$ from $4.9-8.1 \mathrm{MeV}$ have been measured. The new experimental information is used to reliably predict the neutron capture cross section of ${ }^{205} \mathrm{~Pb}$, an important branch point nucleus along the s-process path of nucleosynthesis.
\end{abstract}

\section{Introduction}

Extinct ${ }^{205} \mathrm{~Pb}$ isotopes can be used as powerful cosmochemical tracers in the early solar system [1]. The nuclide ${ }^{205} \mathrm{~Pb}$, which decays to ${ }^{205} \mathrm{Tl}$ with a half-life of 15.3 Myr is of particular astrophysical importance as it is the only short-lived nuclide produced solely by $s$-process nucleosynthesis. As can be seen in Fig. $1,{ }^{205} \mathrm{~Pb}$ is screened from the $r$-process by the stable ${ }^{205} \mathrm{Tl}$ isotope and provides highly important chronometric information about the time span between the last $s$-process nucleosynthetic events that have modified the composition of the solar nebula and the formation of solid bodies in the solar system. The abundance of ${ }^{205} \mathrm{~Pb}$ in the early solar system inferred from carbonaceous chondrites data can also be used to assess whether Asymptotic Giant Branch (AGB) stars and massive Wolf-Rayet stars are the most likely sites of the $s$-process [4,5]. However, this investigation remains sensitive to the amount of freshly produced $s$-process material, including the survival of ${ }^{205} \mathrm{~Pb}$ in AGB stars [6] through both neutron capture and weak interaction processes. Thus, one needs to explain the initial absolute $s$-process abundance of stable lead isotopes which depend on the neutron capture rates. At present the value of the cross section used for calculating abundances in stellar models are purely based on theoretical Hauser-Feshbash predictions [7]. There is an upper limit for the ${ }^{205} \mathrm{~Pb}-{ }^{205} \mathrm{Tl}$ abundance ratio of $9 \times 10^{-5}$ from meteorites [8]. However, no experimental information is available. Due to its astrophysical importance, the Maxwellian-averaged capture cross section on ${ }^{205} \mathrm{~Pb}$ needs to be known accurately, and if not measurable directly, it should at least be experimentally constrained. Accurate capture cross-section measurements on branching-point nuclei such as ${ }^{205} \mathrm{~Pb}$ and ${ }^{204} \mathrm{Tl}$ could be used to provide information on the mean neutron flux during the $s$ process.

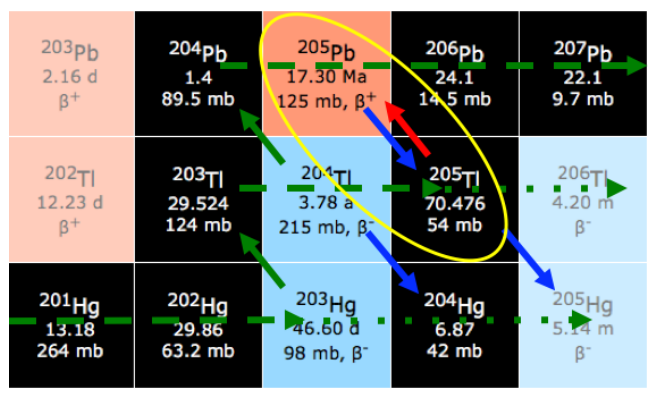

Fig. 1. $s$-process path throughout the thallium-lead isotopes. The green arrows show the s-process path, while the blue and red arrows show $\beta^{-}$and $\beta^{+}$decays respectively.

\footnotetext{
* Corresponding author: tonchev2@llnl.gov
} 
Furthermore, the presence of additional Pygmy Dipole Resonance (PDR) strength below and near the neutron separation threshold has an impact on neutron-capture rates of astrophysical importance $[9,10]$. These $J=1^{-}$ states forming the PDR act as wide open doorway states in the neutron capture process. The short lifetimes of these states permit for fast depopulation of the capture levels towards lower-lying bound states, and stabilize the newly formed nucleus against subsequent neutron emission. Applying detailed balance in the photon bath formed in astrophysical $r$-process event suggests the PDR levels near the neutron threshold will enhance the reverse process of $(\gamma, n)$ photodissociation.

\section{Experimental technique}

The Nuclear Resonance Fluorescence (NRF) technique [11] was used to measure the nuclear dipole response of ${ }^{206} \mathrm{~Pb}$ at the High-Intensity Gamma-Ray Source (HIGS) [12]. Linearly-polarized photons were produced via Compton backscattering of a relativistic electrons from free-electron laser photons. The resultant high intensity photon beam travels $53 \mathrm{~m}$ in a high-vacuum tube before passing through a lead collimator of length $30.5 \mathrm{~cm}$ with a cylindrical hole of $1.27 \mathrm{~cm}$ diameter located $4 \mathrm{~m}$ upstream of the ${ }^{206} \mathrm{~Pb}$ target. The energy distribution of the photon flux was measured with a large volume High Purity Germanium (HPGe) detector, of efficiency $123 \%$ relative to a $7.62 \mathrm{~cm} \mathrm{x} 7.62 \mathrm{~cm} \mathrm{NaI}$ scintillator, placed in the incident beam. For this measurement, the beam was attenuated by copper absorbers mounted upstream. The true energy spectrum from $6500 \mathrm{keV}$ incident photon beam, shown in green, is given in Fig. 2 (a). This spectrum was obtained after removing the response of the HPGe detector (yellow) $[13,16]$. The energy range of interest was scanned, with beam centroid energies, incrementing in steps of approximately the FWHM of the beam ( 200 $\mathrm{keV}$ ), from $4.8 \mathrm{MeV}$ up to $8.3 \mathrm{MeV}$. These spectra are shown in Fig. 2 (b).

The sample was $4000 \mathrm{mg}$ of $99.3 \%$ enriched ${ }^{206} \mathrm{~Pb}$ metal powder. The scattered rays from the lead target were measured by four HPGe detectors, with $60 \%$ relative efficiency, positioned around the ${ }^{206} \mathrm{~Pb}$ target at $(\theta, \varphi)=$ $\left(90^{\circ}, 0^{\circ}\right),\left(90^{\circ}, 90^{\circ}\right),\left(90^{\circ}, 180^{\circ}\right)$, and $\left(90^{\circ}, 270^{\circ}\right)$, where $\theta$ is the polar angle with respect to the horizontally polarized incoming photon beam and $\varphi$ is the azimuthal angle measured from the polarization plane. A fifth detector, of $25 \%$, relative efficiency was placed at $(\theta, \varphi)=$ $\left(135^{\circ}, 0^{\circ}\right)$ to distinguish the spins of positive parity states. The distance between the center of the target to the front surface of the $90^{\circ}$ detectors was $10 \mathrm{~cm}$. All detectors had passive shielding consisting of $3 \mathrm{~mm}$ copper and $2 \mathrm{~cm}$ thick lead cylinders. Lead and copper absorbers of thickness 5 and $3 \mathrm{~mm}$, respectively, covered the openings of the detectors to reduce the low-energy part of the scattered spectrum. The absolute photopeak efficiencies of the HPGe detectors were measured using a set of $\gamma$-ray sources placed in the target position. The measured efficiency was also simulated using MCNP6 [17, 49].

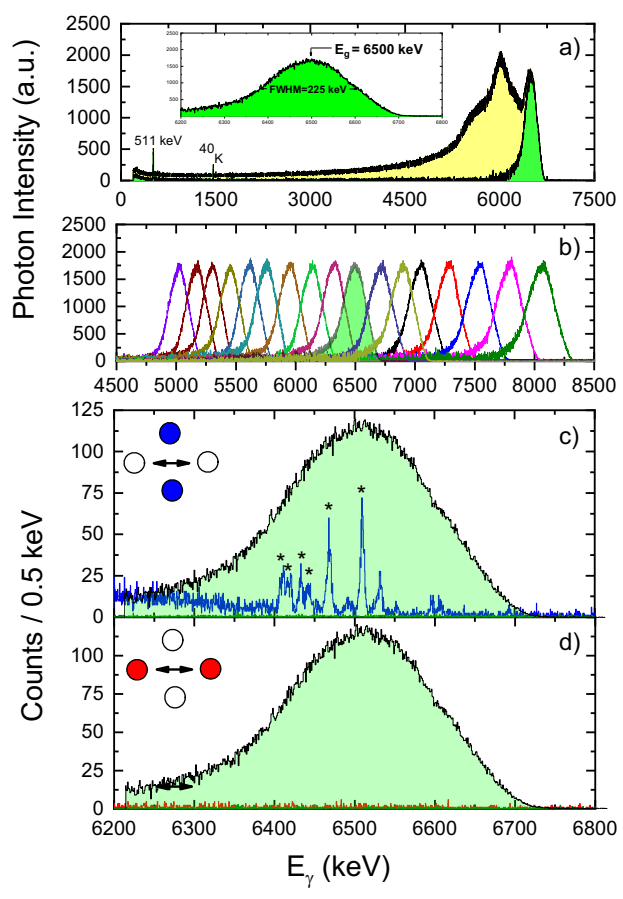

Fig. 2. (a) A photon beam with energy peaked at $6500 \mathrm{keV}$ generated the $\gamma$-ray spectra. (b) The photon energy spectra from all beam energies used in this experiment. (c) The $90 \mathrm{up} /$ down spectra, indicating E1 transitions. (d) The 90 left/right spectra, indicating possible M1 transitions.

\section{Results}

The primary results from photon scattering on ${ }^{206} \mathrm{~Pb}$ was published in Ref. [18]. To calculate the partial decay width $\Gamma_{0}^{x}$ for the newly observed state we compare to the partial decay width of the known states, $\Gamma_{0}^{r}$, observed in Refs. [14, 15], by:

$$
\Gamma_{0}^{x}=\frac{N^{x} F^{r}\left(E_{\gamma}^{x}\right)^{2}}{N^{r} F^{x}\left(E_{\gamma}^{r}\right)^{2}} \Gamma_{0}^{r}
$$

where $N^{x}\left(N^{r}\right)$ is the observed number of counts in an elastic peak in the unknown (reference) state, corrected for the detector efficiency and, if necessary, resonant selfabsorption. The normalization factor $F^{x}\left(F^{r}\right)$ depends on the unknown (reference) states position in the beam energy distribution, corresponding to the relative flux while $E_{\gamma}^{x}\left(E_{\gamma}^{r}\right)$ are the transition $\gamma$-ray energy for the unknown (reference) state. This method was validated using ${ }^{11} \mathrm{~B}$ resonance states [16]. A portion of the of NRF $\gamma$ ray spectra from the photon beam with peak energy of $6500 \mathrm{keV}$ is presented in Fig. 2 (c) and (d). In this particular energy interval, the $\gamma$-ray transitions are only 
steaming from the vertical detectors, showing unambiguous distinction between E1 and M1 transitions $[21,29]$. More detailed information about the NRF technique used at the HIGS facility can be found in Refs. $[19,20,22-31]$.

With higher photon fluxes available at the HIGS facility over the entire energy range, many new states of interest were observed comprised to Refs. [14, 15]. For example, in Fig. 2 (c) the know-resonance states around $6.5 \mathrm{MeV}$ excitation energy from Refs. $[14,15]$ are labeled with stars. Hence, some relatively weak transitions (with $\Gamma_{0}^{x}<100 \mathrm{meV}$ ) are left undetected in the bremsstrahlung NRF experiments. One hundred $1^{-}$states were observed. Twenty-six M1 excited states were also measured. However, it is clear that in this energy region the dipole response is predominantly electric in nature. The M1 strength is localized in two groups around 6.0 and 7.5 $\mathrm{MeV}$ which may be attributed to the M1 spin- resonance $[29,30]$.

The measured elastic, inelastic, and total photoabsorption cross sections of ${ }^{206} \mathrm{~Pb}$, integrated over $250 \mathrm{keV}$ bins, are shown in Fig. 3. As can be seen for excitation energies lower than $\sim 6 \mathrm{MeV}$ the total photoabsorption cross section is determined by the elastic cross section. Near the neutron separation energy of $8.1 \mathrm{MeV}$, the elastic and inelastic cross sections become equal. There is a sudden drop of the elastic (and the corresponding total) cross section at about $6.25 \mathrm{MeV}$.

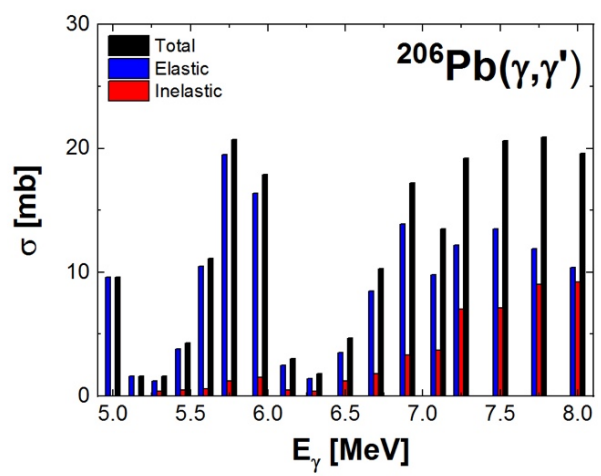

Fig. 3. Elastic, inelastic, and total photoabsorption cross section of ${ }^{206} \mathrm{~Pb}$ below the one-neutron separation energy $\left(\mathrm{S}_{\mathrm{n}}=8.1 \mathrm{MeV}\right)$ averaged over $250 \mathrm{keV}$ energy bins.

\section{Astrophysical relevance}

In order to constrain the capture cross section on ${ }^{205} \mathrm{~Pb}$ we carried out statistical model calculations using the TALYS-1.8 code [32]. The results are shown in Fig. 4 a) where the "Recommended" curve corresponds to the radiative cross section obtained with the presently measured E1 and M1 $\gamma$-ray strength, complemented by the energy-density functional plus quasiparticle-phonon model (EDF+QPM) predictions outside the experimental energy range. As shown in Fig. 4 (a), the QPM supplemented with the QRPA calculations lead to a cross section in excellent agreement with the experimentally constrained recommended one. The uncertainty band in Fig. 4 (a) stems from the experimental uncertainties associated with the $\gamma$-ray strength but also the use of different nuclear level density models [33, 34]. The recommended cross section was obtained with the combinatorial model of Ref. [35]. Note that level densities are constrained by the cumulative number of low-lying levels, including the $J^{\pi}=1$ states, reliably measured in the present experiment up to about $6.5 \mathrm{MeV}$. The contribution of the M1 strength as well as the PDR plus core polarization contributions to the cross section is illustrated in Fig. 4 (b), where the M1 component is seen to be rather insignificant (less than 5\%), but the PDR plus core polarization contributions are crucial for a proper crosssection description.

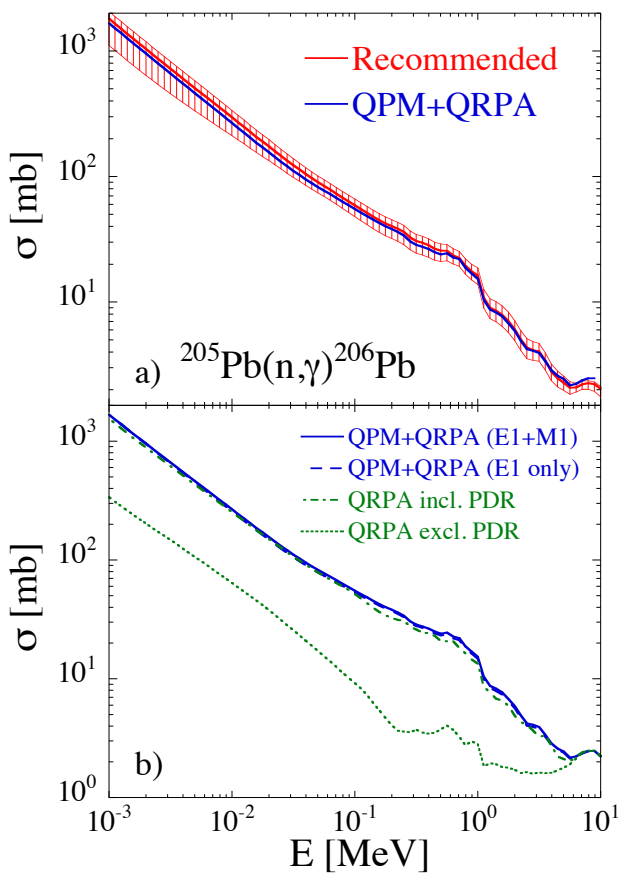

Fig. 4. (a) Radiative capture cross section ${ }^{205} \mathrm{~Pb}(\mathrm{n}, \gamma){ }^{206} \mathrm{~Pb}$ using as input the experimental E1 and M1 dipole strength (red curve) and three-phonon EDF+QPM plus EDF+QRPA predictions (blue curve). (b) Same (a) but now assessing the impact of the various energy regions and multipolarities to the EDF+QPM plus EDF+QRPA calculations. See text for further details.

The experimentally constrained, Maxwellian-averaged cross section at $30 \mathrm{keV}$ is estimated to be $130 \pm 25 \mathrm{mb}$, close to the theoretical value of $125 \pm 22 \mathrm{mb}$ recommended in Ref. [36] and traditionally used in s-process calculations. With this updated cross section, the AGB origin of ${ }^{205} \mathrm{~Pb}$ on the basis of its early solar system abundance and present nucleosynthesis models can be put on more solid ground, despite the uncertainties still 
affecting the distraction of ${ }^{205} \mathrm{~Pb}$ by weak processes and the $s$-process model in AGB stars.

\section{Summary}

In conclusion, experimental high-resolution studies of the PDR mode and EDF+ QPM predictions of the electromagnetic response of ${ }^{206} \mathrm{~Pb}$ permit separation of the PDR from the tail of the GDR and multi-phonon excitations due to core polarization effects. Our findings suggest that the low-energy dipole strength is predominantly electric in character and mainly due to a PDR skin oscillation. However, a substantial contribution from both the low-energy tail of the GDR and multiphonon states to the total E1 strength responsible for the fragmentation pattern of low-energy dipole states is also observed. Moreover, the EDF+QPM theory successfully reproduces the low-energy M1 spectral distribution, suggesting that this distribution is mostly due to spin-flip excitations.

In the context of stellar nucleosynthesis, an updated, experimentally-constrained, Maxwellian-averaged radiative capture cross section for the ${ }^{205} \mathrm{~Pb}(\mathrm{n}, \gamma){ }^{206} \mathrm{~Pb}$ reaction is obtained. The study illustrates the vital and ever increasing role that measurements of exotic modes of excitation in neutron-rich nuclei are playing in the determination of observables of critical astrophysical importance.

\section{Acknowledgments}

This work was performed under the auspices of US DOE by LLNL under contract DE-AC52-07NA27344 and the DOE grants DE-FG02-97ER41033, DE-FG0297ER41041, DE-FG02-97ER41042, DE-FG52 06NA26155, DFG grant Le439/6, and the U.S. Department of Energy, Office of Nuclear Physics, under Grant No. DE-FG02- ER41033.

\section{References}

1. C. Iliadis. Nuclear Physics of Starts. Wiley-VCH Verlag GmbH\&Co. KGaA (2015).

2. S.G. Nielsen, M., Rehkämper, A.N. Halliday, Geochim. Cosmochim. Acta 70, 2643 (2006).

3. R.G.A. Baker, M. Schönbächler, M. Rehkämper, H.M. Williams, A.N. Halliday, Earth Planet. Sci. Lett. 291, 39 (2010).

4. M. Arnould, G. Paulus, G. Meynet, Astron. Astrophys. 321, 452 (1997).

5. G.J. Wasserburg, M. Busso, R. Gallino, K.M. Nollett, Nucl. Phys. A 777, 5 (2006).

6. N, Mowlavi, S. Goriely, M. Arnould, Astron. Astrophys. 330, 206 (1985).

7. KADoNiS website at http://www.kadonis.org.

8. K. Yokoi et al., Astron. Astrophys. 145, 339 (1985).
9. S. Goriely, Phys. Lett. B 436, 10 (1998).

10. N. Tsoneva et. al., Phys. Rev. C 91, 044319 (2015).

11. U. Kneissl et al., Prog. Part. Nucl. Phys. 34, 285 (1995).

12. H. Weller et al., Prog. Part. Nucl. Phys. 62, 257 (2009).

13. C. Sun, Y. K.Wu, J. Li, G. Rusev, and A. P. Tonchev, Phys. Rev. Spec. Top. 12, 062801 (2009).

14. T. Chapuran, R. Vodhanel, M.K. Brusssel, Phys. Rev. C 2, 1420 (1980).

15. J. Enders et al., Nucl. Phys. A 741, 3 (2004).

16. G. Rusev et al., Phys. Rev. C 79, 061302 (2009).

17. S. Carson et al. Nuc. Instr. Meth. Phys. Res. A 618, 190 (2010).

18. A.P. Tonchev et al., Phys. Lett. B 773, 20 (2017).

19. R. Massarczyk et al., Phys. Rev. Lett. 112, 072501 (2014).

20. N. Pietralla et al., Phys. Rev. Lett. 88, 012502 (2002).

21. A.P. Tonchev et al., Phys. Rev. Lett. 104, 072501 (2010).

22. P.M. Goddard et al., Phys. Rev. C 88, 064308 (2013).

23. C. Romig et al., Phys. Rev. C 88, 044331 (2013).

24. M. Scheck et al., Phys. Rev. C 87, 051304(R) (2013).

25. R. Schwengner at al., Phys. Rev. C. 87, 024306 (2013).

26. S. Volz et al., Nucl. Phys. A 779, 1 (2006).

27. H. Ohgaki, et al. Nucl. Phys. A 649, 73c (1999).

28. R. Schwengner et al., Phys. Rev. C 78, 064314 (2008).

29. G. Rusev et al., Phys. Rev. Lett. 104, 072501 (2013).

30. Krishichayan et al., Phys. Rev. C 91, 044328 (2015).

31. B. Leher et al., Phys. Lett. B 756, 72 (2016).

32. A.J. Koning, D. Rochman, Nuclear Data Sheets 113, 2841 (2012).

33. A.J. Koning, S. Hilaire, S. Goriely, Nucl. Phys. A 810, 13 (2008).

34. R. Capote, M. Herman, P. Oblozinsky, et al., Nuclear Data Sheets 110, 3107 (2009).

35. S. Goriely, S. Hilaire, and A.J. Koning, Phys. Rev. C 78, 064307 (2008)

36. Z. Y. Bao et al., Atomic Data and Nuclear Data Tables 76, 70 (2000). 